\begin{tabular}{|c|c|c|}
\hline & Int.J.Curr.Microbiol.App.Sci (2016) 5(11): 906-911 & \\
\hline & International Journal of Current Microbiology and Applied Sciences & morken - \\
\hline & ISSN: 2319-7706 Volume 5 Number 11 (2016) pp. 906-911 & \\
\hline $\begin{array}{l}\text { EXCELLENT } \\
\text { PUBLISHERS }\end{array}$ & & (1) \\
\hline
\end{tabular}

Original Research Article

http://dx.doi.org/10.20546/ijcmas.2016.511.104

\title{
Prevalence of Oral Lesions in Adult Diabetic Patients referred to Dentistry Faculty of Tabriz University of Medical Sciences between 2009-2014
}

\author{
Ali Barzegar ${ }^{1}$, Parya Emamverdizadeh ${ }^{2 *}$ and Yaser Hemmati $^{3}$ \\ ${ }^{1}$ Assistant Professor, Department of Nutrition in Community, Faculty of Nutrition , \\ Tabriz University of Medical Sciences, Tabriz, Iran \\ ${ }^{2}$ Assistant Professor, Department of Oral and Maxillofacial Pathology, Faculty of Dentistry, \\ Tabriz University of Medical Sciences, Tabriz, Iran \\ ${ }^{3}$ Resident of Prosthodontics, Faculty of Dentistry, Tabriz University of Medical Sciences, \\ Tabriz, Iran \\ *Corresponding author
}

\begin{abstract}
A B S T R A C T
Diabetes cause various complications that lead to complicated mechanism of action, many of these complications are still unknown, understanding these effects and preventing them can be very helpful for planning to deal with diabetes. Therefore, regarding the need to have more information on this disease and its

\section{Keywords}

Diabetes,

Complications, Prevalence.

Article Info

Accepted:

25 November 2016

Available Online:

10 December 2016 complications, and the contradicted results of previous studies on oral effects of diabetes on the other hand, we conducted this study on prevalence of oral lesions in adult diabetic patients referred to dentistry faculty of Tabriz University of medical science between 2009-2014. Subjects in this descriptive study includes adult diabetic patients referred to dental faculty of Tabriz University of medical science between 2009-2014. Conducted data from this study were analyzed by using SPSS15 statistical software and descriptive statistic methods (frequency). The results showed that the prevalence of majority of invested oral lesions including periodontal, soft tissue, precancerous and tumoral lesions in diabetic patients is more than healthy people. Regarding the high prevalence of all investigated oral lesions except oral candidiasis, geographic tongue and aphthous in diabetic patients in comparison with healthy people, a close cooperation between patients, the doctors and dentists is needed to enhance general and oral health condition of diabetic patients.
\end{abstract}

\section{Introduction}

DM is a syndrome in which energy balance and metabolism is disturbed due to lack of insulin secretion or its malfunction, resulting in abnormal metabolism of carbohydrates, protein and fat (Khozeimeh et al., 2005). In general, diabetes mellitus is divided into diabetes type I, diabetes type II, gestational diabetes and other specific types (Little et al., 2012). Diabetes mellitus (DM) has a high prevalence rate, so that in a study, the infection rate worldwide in 2000 was estimated $2.8 \%$ which according the predictions, will be around $4.4 \%$ in 2030 (Wild et al., 2004). In Iran, the prevalence 
rate in 2005 was estimated 3.8 million out of 68 million people (Azizi, 2005). DM imposes a very heavy costs on society, so that during 1992 in the US, 85 to 92 billion dollars was spent treating these patients (Javitt et al., 1995). This disease causes symptoms such as loss of vision, kidney failure, amputation, stroke and coronary artery stenosis (Leung et al., 1994).

The DM causes oral lesions such as periodontal lesions, lesions associated with Candida, soft tissue lesions including: irritation fibroma, traumatic ulcers and aphtha. Pre-cancerous lesions include: erythroplakia, leukoplakia, Lichen Planus, tumoral lesions divided into two categories: benign and malignant lesions (Saini et al., 2010; Ujpál et al., 2004).

Due to the importance of this issue, many investigations have been conducted in this regard. It was observed in a study that the prevalence of the oral lesions, including denture stomatitis and angular chelitis in diabetics is higher than in people without diabetes; however, no relationship was observed between diabetes mellitus and precancerous lesions. In another study comparing patients with controlled and uncontrolled diabetes mellitus, the prevalence of candidiasis and inflammatory proliferative lesions were higher in patients with uncontrolled diabetes (Quirino et al., 1995). As well in another study, the prevalence of oral Lichen Planus (a precancerous lesion) has been observed in patients with diabetes, especially type I, which can indicate the relationship between diabetes with an autoimmune phenomenon (Petrou-Amerikanou et al., 19998). In the study by Ujpal et al., the prevalence of benign and malignant oral tumors in diabetic patients was higher than in healthy control group. Diabetes has many complications due to the complexity of the mechanism of disease, yet many of its effects are unknown.
Recognition of these complications and the prevention approaches can be very useful for planning to deal with diabetes. The prevalence of this disease in our country is very high and imposes heavy costs and many problems to the society. Therefore, considering the need to learn more about this disease and its complications and the existence of controversies in the other articles in the field of oral complications of the disease, this study was conducted in to investigate the prevalence of oral lesions in diabetic patients referred to the Faculty of Dentistry, Tabriz University of Medical Sciences between the years 2009-2014.

\section{Materials and Methods}

In a case-control study in 2015 in Tabriz on adult diabetic patients referred to the Faculty of Dentistry, Tabriz University of Medical Sciences, the prevalence of oral lesions was examined in these patients. The case group included the adult diabetic patients referred to the Faculty of Dentistry, Tabriz University of Medical Sciences with no other systemic disease, and the age older than 19 years. The control group included the healthy individuals older than 19 years. In this study, all documents contained in the archives of the Faculty of Dentistry since 2009 to 2014 were assessed by the researcher in a thorough census and information on each patient such as age, sex and oral lesions were recorded in the checklist.

\section{Ethical Considerations}

Since no therapeutic intervention occurred in this study, there was no ethical consideration necessary, and all patient information was confidential.

\section{Results and Discussion}

Out of 38,000 of total cases under study, 187 patients $(0.47 \%)$ had diabetes. Also, 346 
cases were healthy people under study. The mean age of the patients was51.8 \pm 11.6 years. And the mean age of male and female patients was $54.9 \pm 11.3$ and $49.8 \pm 11.4$ years respectively.

Frequency (\%) of types of lesions is listed in Table 1, indicating that periodontal lesions were the most common lesion in both groups.

Out of the four candidiasis lesions in healthy individuals, two cases were observed in the corners of the lips. Among the patients, only one candidiasis lesion was reported in the corners of the mouth.

Out of 5 cases of fibrous lesion (a soft tissue lesion) in healthy individuals, 4 cases were observed in the buccal mucosa. Out of 9 cases of fibrous lesion in patients, 5 cases were observed in the buccal mucosa and 2 at the tip of the tongue.

Both of the two traumatic ulcers (a soft tissue lesion) found in patients were in the area of tongue.

The area of aphtha injury (a soft tissue lesion) in both groups was labial mucosa.

In both groups, one case of leukoplakia lesion was reported that occurred in the sides of the tongue in healthy individuals and in retromolar pads the area in patients.

One case of erythroplakia lesion in patients was occurred in retromolar pad area.

The most common area of Lichen Planus lesions (a pre-cancerous lesion) was the buccal mucosa in patients (7 of 9 lesions) and healthy individuals (5 of 6 lesions).

The area of the one case of benign tumoral lesion in healthy individuals was the lower lip, and the area of the one case of benign tumoral lesion the in patients was labial mucosa of the upper jaw.

The area of the one case of malignant lesion in healthy individuals was in the tongue border and the area of the one case of malignant lesion in patients was the dorsal surface of the tongue.

Diabetes has a wide association with oral demonstrations, however, many contradictions can be observed about the importance of diabetes as a risk factor for oral lesions in the studies reported around the world (Sandberg et al., 2008). Guggenheimer et al., reported that differences in the prevalence of oral lesions are probably due to different physiological response to the behavior of types of diabetes. Other possible effective factors are the differences in blood glucose control, disease duration and age of the patients under study.

These differences may also be due to differences in statistical methodology and the sample size under study. In addition, in some studies, diagnosis of DM may be based on blood glucose readings lower than the amount declared by WHO.

In this study, 178 of the people under study (38.2\% male) were diabetic and $346(42.8 \%$ male) were healthy.

In this study, the mean age of patients and control subjects was 51.8 \pm 11.6 and $35.5 \pm 11.6$ years respectively.

In this study, the prevalence of periodontal lesions was $54.2 \%$ in diabetic patients and $33.5 \%$ in healthy individuals, where a higher prevalence rate was observed in diabetic patients possibly due to changes in host response, collagen metabolism and cardiovascular status of the patients. In addition, in patients with uncontrolled 
diabetes, severe inflammatory response to the bacteria can be observed, which, along with the tissue repair process, may be effective in the process of inflammatory response and destruction of periodontal tissues (Collin et al., 1998).

Similar to the results obtained in this study, another study demonstrated that the prevalence of periodontal lesions in diabetic patients was higher than in healthy individuals and unlike this study, in a study by Collin, there was no clear relationship between periodontal lesions and diabetes.

According to this study, the prevalence of lesions associated with Candida in diabetic patients and healthy individuals were not much different (respectively 4 out of 346 $(1.16 \%)$ and 1 out of 178 patients $(0.56 \%)$ ). Similar to this study, in the study by BudtzJorgen, the prevalence of candidiasis lesions in the two groups of healthy and diabetic people was nearly similar (Budtz-jorgensen et al., 1970).

In this study, the prevalence of soft tissue lesions was $6.7 \%$ of 178 people in diabetic patients and $2.6 \%$ of 346 people in the healthy controls, which indicates a higher percentage among diabetics. Also in the study by Khozeimeh-Mohseni, the higher prevalence of lesions has been verified.

Unlike most oral lesions, the prevalence of geographic tongue in healthy individuals $(1.16 \%)$ was higher than diabetic patients $(0 \%)$. In the study by Costa et al., no differences were observed in linguistic abnormalities between healthy and diabetic patients. However, in the study by Rajansaini et al., the prevalence of geographic tongue in diabetic patients was significantly higher than in healthy patients.

The prevalence of fibroma in diabetic patients $50.50 \%$ of 178 people, indicating a higher prevalence than in healthy controls (1.44\% of 346 people). The incidence of traumatic ulcers in diabetic patients was higher. In Guggenheimer's study, stimulatory fibroma and traumatic ulcers in diabetic patients have been observed more than in the control group (Guggenheimer et al., 2000).

The higher incidence of stimulatory fibroma and traumatic ulcers in the mouth of diabetic patients is influenced by factors such as changes in the oral environmental condition, the loss of some or all teeth and use of prosthesis by patients, smoking, alcohol, delayed healing, increasing age and duration of diabetes. However, in the study by Khozeimeh and Mohseni, no statistically significant difference was observed in both lesions of traumatic ulcer and stimulatory fibroma.

During this study, the prevalence of aphtha in diabetic patients $(0.56 \%)$ was similar to healthy individuals $(0.58 \%)$. However, in the study by Saini et al., the prevalence of the aphtha in diabetic patients was higher than in healthy people.

In this study, the prevalence of precancerous lesions of leukoplakia and erythroplakia in diabetic patients was $1.12 \%$ of 178 people versus $0.28 \%$ of 346 in healthy individuals, which indicates a higher prevalence in diabetic patients. However, studies show large controversies in relation to this issue.

It seems that diabetes-induced atrophic and inflammatory oral demonstrations may be associated with neoplastic changes. In addition, higher prevalence of candidiasis lesions in diabetic patients showed the greater tendency of squamous cells to reproduction and metaplasia in these patients. As well, in the study of Marta Ujpal, pre-cancerous lesions in diabetic 
patients were more prevalent than in healthy people. However, in the study of Saini et al., there was no significant difference between the two groups

Table.1 Frequency(\%) of several lesions between two groups

\begin{tabular}{lcc}
\hline & Healthy People & $\begin{array}{c}\text { Diabetic } \\
\text { People }\end{array}$ \\
\hline $\begin{array}{l}\text { Periodontal Lesions } \\
\text { Candida Lesions }\end{array}$ & $\begin{array}{c}\text { Soft Tissue Lesions } \\
\text { 4(1.16\%) }\end{array}$ & $\begin{array}{c}90(54.2 \%) \\
1(0.56 \%)\end{array}$ \\
\hline Geographic tongue & $4(1.16 \%)$ & 0 \\
Fibroma & $5(1.44 \%)$ & $9(5.05 \%)$ \\
Traumatic Ulcer & 0 & $2(1.12 \%)$ \\
Aphtha & $2(0.58 \%)$ & $1(0.56 \%)$ \\
Total & $9(2.6 \%)$ & $2(6.7 \%)$ \\
\hline \multicolumn{3}{c}{ Pre-cancerous Lesions } \\
\hline Leukoplakia & $1(0.28 \%)$ \\
Erythroplakia & 0 & $1(0.56 \%)$ \\
Lichen Planus & $6(1.73 \%)$ & $1(0.56 \%)$ \\
Total & $7(2.02 \%)$ & $9(5.05 \%)$ \\
\hline \multicolumn{3}{c}{$11(6.17 \%)$} \\
\hline Benign & Tumoral Lesions \\
Malignant & $1(0.28 \%)$ & $1(0.56 \%)$ \\
Total & $1(0.28 \%)$ & $1(0.56 \%)$ \\
\hline
\end{tabular}

In this study, the prevalence of Lichen Planus (a pre-cancerous lesion) in diabetic patients was approximately $6.17 \%$ of 178 people, which indicates a significant difference compared to healthy individuals with the prevalence of $2.02 \%$. In the study of Saini et al., the prevalence of Lichen Planus in diabetic patients was higher than in healthy controls. Although in some articles, the authors have suggested lower prevalence of OLP in diabetic patients. For example, in the study of Borghelli et al., the prevalence of OPL in diabetic patients has been reported $0.55 \%$ of 729 diabetic patients vs. $0.74 \%$ of 676 healthy individuals.

In this study, the prevalence of tumoral lesions is $1.12 \%$ of 178 diabetic patients vs. $0.58 \%$ of 346 healthy individuals, indicating a higher prevalence in diabetic patients. A similar study by Ujpal in Hungary showed that the prevalence of benign tumors and malignant lesions among diabetic patients studied was significantly higher than healthy people. In other studies, as well, the relationship between DM and many types of cancers, including oral cancers has been emphasized.

In conclusion, given that in this study, the prevalence of all oral lesions evaluated, except for candidiasis lesions, geographic tongue and aphtha in diabetic patients is higher than in healthy individuals, a close collaboration between patients, doctors and dentists is required to enhance and improve the general and oral health of diabetic patients.

\section{References}

Al-Maskari, A.Y., Al-Maskari, M.Y., AlSoudi, S. 2011. Oral manifestations and 
complications of diabetes mellitus: a review. Sultan Qaboos University Med. J., 11(2), 179.

Azizi, F. 2005. Diabetes care and prevention in Iran. Diabetes Voice, 50(4), 15-17.

Budtz-jorgensen, E., Bertran, V. 1970. Denture Stomatitis: the Etiology in Trauma and Infection. Acta Odont Scand, 28, 71-90.

Collin, H.L., Uusitupa, M., Niskanen, L., Kontturi-Narhi, V., Markanen, H., Koivisto, A.M., et al. 1998. Periodontal Findings in Elderly Patients with Noninsulin Dependent Diabetes Mellitus. $J$. periodontol., 9, 962-966.

Guggenheimer, J., Moore, P.A., Rossie, K., Myers, D., Mongelluzo, M.B., Block, H.M., Weyant, R., et al. 2000. Insulindependent diabetes mellitus and oral soft tissue Pathologies Prevalence and characteristics of non-candidal lesions. Oral Surgery Oral Medicine Oral Pathol., 89(5), 570-6.

Javitt, J.C., Chiang, Y. 1995. Economic impact of diabetes. Diabetes in America, 601-611.

Khozeimeh, F., Mohseni, G. 2005. Oral Appearances in Patients with Diabetic Type I. J. Isfahan Dental School, 1(2);27-30. (Persian)

Leite, R.S,. Marlow, N.M., Fernandes, J.K. 2013. Oral Health and Type 2 Diabetes. NIH public access, 345(4), 271-273.

Leung, G., Lam, K. 1994. Diabetic complications and their implications on health. Diabetes care, 17, 1190-1192.

Little, J.W., Falace, D.A., miller, C.S., Rhodus, N.L. 2012. Dental management of the medically compromised patient,8thed.Elsevier, Missouri, 220.
Petrou-Amerikanou, C., et al. 1998. Prevalence of oral lichen planus in diabetes mellitus according to the type of diabetes. Oral dis., 4(1), 37-40.

Quirino, M., Birman, E.G., Paula, C.R. 1995. Oral manifestations of diabetes mellitus in controlled and uncontrolled patients. Braz. Dent. J., 6(2), 131-136.

Saini, R., Al-Maweri, S.A, Saini, D., Ismail, N.M., Ismail, A.R. 2010. Oral mucosal lesions in non-oral habit diabetic patients and association of diabetes mellitus with oral precancerous lesions. Diabetes research and clinical practice, 89(3), 320-326.

Sandberg, G.E., Sundberg, H.E., Fjellstorm, C.A., Wikbald, F.K. 2000. Type 2 diabetes and oral health:A comparison between diabetic and non-diabetic subjects. Diabetic research and clinical practice, 50, 27-34.

Ujpál, M., Matos, O., Bíbok, G., Somogyi, A,. SzabóG, Suba, Z. 2004. Diabetes and Oral Tumors in Hungary Epidemiological correlations. Diabetes care, 27, 770-4.

Vasconcelos, B.C., Novaes, M., Sandrini, F.L., Fabio, A.W., Coimbra, L.S. 2008. Prevalence of Oral Lesions in Diabetics: a Preliminary Study. Brazilian J. Otorhinolaryngol., 74(3), 423-8.

Wild, S., et al. 2004. Global prevalence of diabetes estimates for the year 2000 and projections for 2030. Diabetes care, 27(5): 1047-1053.

Wysocki, G.P., Daley, T.D. 1987. Benign migratory glossitis in patients with juvenile diabetes. Oral Surgery, Oral Medicine, Oral Pathol., 63(1), 68-70.

\section{How to cite this article:}

Ali Barzegar, Parya Emamverdizadeh and Yaser Hemmati. 2016. Prevalence of Oral Lesions in Adult Diabetic Patients referred to Dentistry Faculty of Tabriz University of Medical Sciences between 2009-2014. Int.J.Curr.Microbiol.App.Sci. 5(11): 906-911.

doi: http://dx.doi.org/10.20546/ijcmas.2016.511.104 